\title{
MedienPädagogik
}

Zeitschrift für Theorie und Praxis der Medienbildung

Themenheft Nr. 32: Offenheit in Lehre und Forschung - Königsweg oder Sackgasse? Herausgegeben von Franziska Bellinger und Anna Heudorfer

\section{Eine offene Bildungsressource (OER) ist konsequent eingesetzt eine Chance für den Hochschulzugang}

\section{Ein Praxisbericht}

Anja Lorenz

\begin{abstract}
Zusammenfassung
Im Bereich der akademischen Weiterbildung werden auch zunehmend offene Online-Kurse (MOOCs) für Hochschulen interessant. An der Technischen Hochschule Lübeck werden im Rahmen des Projekts pMOOCs insb. Berufstätige angesprochen und mögliche Übergänge in die Hochschule untersucht. In diesem Beitrag wird der MOOC «Netzwerksicherheit» in seiner spezifischen Gestaltung als offene Lernressource (OER) vorgestellt und die Konsequenzen der Öffnung anhand von Nutzungszahlen und Evaluationsergebnissen beleuchtet. Insbesondere die Gewinnung von Teilnehmenden, deren Interaktion mit dem Kurs sowie Rückmeldungen zu einer im Anschluss angebotenen Klausur zum Erwerb eines prinzipiell anrechenbaren Hochschulzertifikats zeigen deutliche Unterschiede zu traditionellen (Online-)Studierenden.
\end{abstract}

Consequently used Open Educational Resources (OER) are an Opportunity for Access to Higher Education: A Case Study.

\begin{abstract}
In the area of further academic training, open online courses (MOOCs) are also becoming increasingly interesting for universities. At the Technische Hochschule Lübeck - University of Applied Sciences, the pMOOCs project addresses professionals and examines open courses as a possible step into the higher education. The article introduces the MOOC "Network Security» in its specific design as an open educational resource (OER). The consequences of opening up are examined on the basis of usage statistics and evaluation results. In particular, MOOC participants show clear differences compared to traditional (online) students in the acquisition of participants, their interaction with the course and feedback on a subsequent exam to obtain a university certificate.
\end{abstract}




\section{Einleitung: Hochschulzugang für Berufstätige}

Die Studiengänge an Hochschulen sind i.d.R. für das grundständige Studium ausgelegt, d.h. die Studierenden widmen sich im Alter von etwa Anfang 20 ihren Lehrveranstaltungen in Vollzeit. Immer mehr werden im Rahmen des lebenslangen Lernens (LLL) auch berufsbegleitendes Studieren und wissenschaftliche Weiterbildung als Aufgaben der Hochschulen erschlossen. Im Bund-Länder-Wettbewerb «Aufstieg durch Bildung: offene Hochschulen» werden Konzeptentwicklungen für diese sog. nicht-traditionellen Studierenden gefördert (BMBF 2018). Diese Zielgruppe wird aufgrund ihrer Motivation, Erwartungen, Vorbildung und Zielen, aber auch durch die Einbindung in Beruf und Familie als stark heterogen beschrieben (Jürgens und Zinn 2015). Für sie steht ausgehend von der bereits vorhandenen Qualifikation die eigene Fortbildung und fachliche (Weiter-) Entwicklung im Vordergrund. So wählen sie selten vollkommen neue Studienfächer, sondern orientieren sich überwiegend an ihrem bisherigen beruflichen Hintergrund. Die grössten Bedenken bestehen neben der Finanzierung des Studiums vor allem darin, ob die eigenen zeitlichen und organisatorischen Ressourcen ausreichen, um die zu erwartenden Mehrfachbelastung «aus Familie, Haushalt, Erwerbstätigkeit und Studium» zu meistern (Jürgens und Zinn 2015, 50).

Ein bisher klassischer Weg der akademischen Weiterbildung für berufstätige Menschen ist das Fernstudium. Aus den besonderen Anforderungen an Workload, Anerkennung extern erbrachter Leistungen, Studienorganisation, sachgemässer Modularisierung, passgenauem Zugang, Prüfungsorganisation sowie Beratungs- und Betreuungsangebote im Fernstudium leitet Czanderle (2017) ab, dass die

«bloße Adaption vorhandener Strukturen an Präsenzhochschulen [...] der Zielgruppe ebenso nicht gerecht werden können, wie die Umstellung von traditionellen Präsenzstudiengängen auf das Fernstudienformat allein durch die Reduzierung der Präsenzzeit.»

Die dabei zu beobachtende Entwicklung, dass für das berufsbegleitende Fernstudium vor allem spezifische Online-Studienangeboten insb. als weiterbildende MasterStudiengänge nachgefragt werden (siehe bspw. Wannemacher 2014), ist daher wenig überraschend.

Ebenso erscheint die Entwicklung der Studiengänge durch auf das Fernstudium spezialisierte Hochschulen, Hochschulbereiche oder Tochtergesellschaften üblich und einer anforderungsgerechten Bereitstellung angemessen. Hierzu zählt auch das Institut für Lerndienstleistungen der Technischen Hochschule Lübeck ${ }^{1}$, das als zentrale Einrichtung die Entwicklung von Studienangeboten realisiert, bzw. mit der 100\%igen Tochter oncampus als Dienstleisterin die hierfür nötigen Infrastruktur für die Virtuelle Fachhochschule (VFH) bereitstellt. Mit dem Projekt pMOOCs wurden hier

1 Zum 01.09.2018 wurde die Fachhochschule Lübeck in Technische Hochschule Lübeck umbenannt. Im Text wird durchgehend die aktuelle Bezeichnung verwendet. 
nun weitere Möglichkeiten durch offene Angebote von Hochschulen erprobt und untersucht.

Der Beitrag stellt im 2. Abschnitt das Projekt pMOOCs im Allgemeinen vor und greift in Abschnitt 3 den offenen Online-Kurs zur Netzwerksicherheit als Fallbeispiel konkret heraus. Exemplarisch werden wichtige Gestaltungselemente insbesondere im Gegensatz zum regulären Online-Studium beschrieben. Schliesslich werden in Abschnitt 4 die Konsequenzen dieser Öffnung anhand des betreuten Kursdurchlaufs vom 29.03. bis 20.06.2016 sowie der nachfolgenden Klausur beschrieben.

\section{Das Projekt pMOOCs}

Das Projekt $p M O O C s^{2}$ der Technischen Hochschule Lübeck zielt darauf ab, die Öffnung der Hochschule gegenüber der Zielgruppe berufstätiger Menschen mithilfe von sechs offenen Online-Kursen (MOOCs) unter verschiedenen Fragestellungen ${ }^{3}$ (insb. zur bedarfsgerechten Gestaltung von Angeboten für diese Zielgruppe, zum Übergang in die Hochschule und der damit verbundenen Anrechnung) zu erproben. Thematisch wurden dabei Fächer aus den Bereichen Medieninformatik, Wirtschaftsingenieurwesen und Entrepreneurship auf Bachelor- und Master-Niveau gewählt:

- Projektmanagement (Ba./M.)

- Netzwerksicherheit (M.)

- Mathematik (Ba./Brückenkurs)

- Entrepreneurship (M.)

- Search-Engine-Marketing (Ba.)

- Kosten- \& Leistungsrechnung (Ba.)

An der Technischen Hochschule Lübeck werden offene Online-Kurse (MOOCs) neben Präsenz- und Online-Studium als eine dritte strategische Säule betrachtet, sodass hier frühzeitig mit diesem Bildungsformat experimentiert und die dafür nötige Infrastruktur aufgebaut wurde (Lorenz et al. 2015). Diese wurden im Projekt verstetigt und erweitert, führten aber auch zu neuen Herausforderungen und Fragestellungen. Am Beispiel des Kurses Netzwerksicherheit (\#nwsMOOC) werden in diesem Beitrag Potenziale und Konsequenzen insbesondere der Öffnung und damit nötigen Skalierbarkeit der Bildungsangebote dargestellt.

2 Gefördert vom BMBF im Rahmen dieses Wettbewerbs «Aufstieg durch Bildung: offene Hochschulen» unter dem Projekttitel "Strategische Implementierung von 'professional Massive Open Online Courses' (pMOOCs) als innovatives Format durchlässigen berufsbegleitenden Studierens», Förderkennzeichen: $160 \mathrm{H} 21016$.

3 Informationen und Ergebnisse zum Projekt auf http://pmooc.oncampus.de/. 


\section{Öffnung einer Lehrveranstaltung am Beispiel \#nwsMOOC}

Der MOOC Netzwerksicherheit (\#nwsMOOC) ${ }^{4}$ wurde zusammen mit Andreas Hanemann von der Technischen Hochschule Lübeck entwickelt und im Zeitraum von zwölf Wochen (29.03. bis 20.06.2016) auf der MOOC-Plattform mooin ${ }^{5}$ erstmalig durchgeführt. Zum Kursende hatte der MOOC 4.099 Teilnehmende.

\section{Inhalte des Kurses}

Die Grundlage bildete dabei das Modul «Sicherheitstechniken in Kommunikationsnetzen» der Virtuellen Fachhochschule, das mit einem Umfang von 5 ECTS bereits im (geschlossenen) Online-Studium eingesetzt wird (siehe Tabelle 1).

\begin{tabular}{|l|l|l|}
\hline Woche & Thema & Dauer \\
\hline 1 & Einführung & 1 Woche \\
\hline 2 & Angriffe aus dem Internet & 3 Wochen \\
\hline 3 & Abwehr von Angriffen & 3 Wochen \\
\hline 4 & Sichere Kommunikation & 3 Wochen \\
\hline 5 & IT-Grundschutz und ISO 27000 & 2 Wochen \\
\hline
\end{tabular}

Tab. 1.: Thematischer Aufbau des nwsMOOCs.

Die Umsetzung des Moduls als offener Online-Kurs umfasste nicht einfach nur die Bereitstellung des bereits vorhandenen interaktiven Online-Skripts über die Plattform. Darstellungsform, Didaktik und damit auch deren technische Umsetzung mussten für ein offenes Kursformat überdacht werden. So wurden die Lerninhalte um $47 \mathrm{Vi}$ deos mit Erläuterungen zu Kernthemen erweitert. Zudem führte Hanemann sechs Interviews mit Expertinnen und Experten aus dem Themenfeld, die ebenfalls im Kurs bereitgestellt wurden und die Praxisnähe der behandelten Inhalte aufzeigen:

- Dr. Mark Yampolskiy (University of South Alabama in Mobile, Alabama): «Sicherheit von Cyber-Physical Systems»

- Olivia von Westernhagen (selbstständige Publizistin): «Malware und Reverse Engineering»

- Alexander von Gernler (genua): «Firewalls und VPNs»

- Dr. Silvia Knittl (accessec GmbH): «Identity and Access Management»

- Ralf Paffrath (DFN): «eduroam»

- Jürgen Brauckmann (DFN-CERT): «PKI und Zertifikate»

4 Online frei zugängig unter https://www.oncampus.de/nwsmooc.

5 mooin wurde im Zuge des Relaunchs von oncampus.de im Dezember 2017 ein Teil der neuen «World of Learning», die neben den MOOCs auch Online-Studiengänge, Weiterbildungs- und Selbstlernangebote anbietet. 


\section{Kompetenzorientierte Überprüfung des Lernerfolgs}

Die Überprüfung des Lernerfolgs musste zudem weitestgehend für eine automatisierte Auswertung aufbereitet werden. Im regulären Online-Modul wird i.d.R. von einer überschaubaren Anzahl von etwa 20 Studierenden ausgegangen, weshalb individuelle Korrekturen (bspw. von Einsendeaufgaben oder anderen kompetenzgerechten Aufgabenformen) durchaus von den betreuenden Personen zu leisten sind. In offenen Online-Kursen ist die Zahl der Teilnehmenden allerdings nicht vorhersehbar. So sind aktuell ${ }^{6}$ für die Kurse im Projekt pMOOCs zwischen 128 und 4780 Menschen angemeldet. Zudem wird nach Ablauf der betreuten Phase die weitere Nutzung als sog. Selbstlernangebot bereits in der Konzeption mit berücksichtigt. Die Aufgabenformate müssen daher im Zweifel auch für eine sehr hohe Teilnehmendenzahl umsetzbar sein, was den hohen Automatisierungsgrad begründet. So wurde das einfache Verständnis der vermittelten Inhalte direkt in den Videos über H5P-Quizzes ${ }^{7}$ abgefragt. Kompetenzorientierte Aufgabenformate wurden mithilfe eines bereitgestellten TestServers entwickelt: praktische Aufgabenstellungen wurden dabei so formalisiert, dass sie sich automatisch überprüfen lassen. Ein Beispiel aus Kapitel 2 Lektion $4^{8}$ lautete:

«Führen Sie einen Port Scan des Servers https://nwsmooc.mooin.org mittels nmap/zenmap durch. Welche Ports sind offen? Bitte markieren Sie die richtigen Portnummern (nur die Zahlen)! TCP Port 2223255380135443 9992.» Die Aufgabe ist nach dem Zufallsprinzip mit 255 möglichen Kombinationen kaum in einer realistischen Zeit von den Teilnehmenden zu erraten. Ein Grossteil der Aufgaben wurde auf diese Weise formuliert.

\section{Dokumentation des Lernerfolgs innerhalb des Online-Kurses}

Sowohl für die Beantwortung der Fragen in den Videos, als auch für die Bearbeitung der Aufgaben wurden ein oder mehrere Badges ausgestellt (siehe dazu auch Tabelle 3). Hierfür werden in mooin, das auf dem Open-Source-LMS moodle ${ }^{9}$ aufsetzt, Badges an den Abschluss von (in diesem Fall H5P-) Aktivitäten geknüpft. Zudem wir automatisch ein Teilnahmezertifikat generiert, wenn über $80 \%$ aller Aktivitäten erfolgreich bearbeitet wurden.

6 Stand: 03.02.2018, wobei die Kurse aufgrund der unterschiedlichen Produktionszeiträume bereits unterschiedlich lange zur Anmeldung online stehen.

7 Hierfür wurde der Content-Typ «Interactive Video» eingesetzt https://h5p.org/interactive-video. Beim ersten Durchlauf kam noch die Quiz-Software Capira zum Einsatz, deren Entwicklung inzwischen eingestellt wurde.

8 Online unter https://www.oncampus.de/course/weiterbildung/moocs/netzwerksicherheit?chapter=2\&sel ected_week $=14$.

9 https://moodle.org. 


\section{Durchführung einer (Präsenz-) Klausur}

Optional konnten die MOOC-Teilnehmenden am 12.07.2016 an einer Präsenzklausur teilnehmen. Dafür wurden die ohnehin stattfindenden Klausuren des regulären Studienmoduls der VFH an fünf Standorten ${ }^{10}$ auch für MOOC-Teilnehmende geöffnet (siehe dazu auch Tabelle 4). Bei erfolgreichem Absolvieren der Klausur wurde den MOOC-Teilnehmenden ein Hochschulzertifikat der Technischen Hochschule Lübeck ausgestellt, das einen Workload von 5 ECTS bescheinigte sowie die im mit dem MOOC erreichten und mittels Klausur überprüften Lernergebnisse auflistet. Dieses Hochschulzertifikat wird innerhalb der Virtuellen Fachhochschule für das entsprechende Studienmodul «Sicherheitstechniken in Kommunikationsnetzen» akzeptiert und angerechnet. An anderen Hochschulen kann eine Anrechnung durch das Prüfungsamt erfolgen, wenn dieses auf Antrag die Lernergebnisse als gleichwertig zu einem entsprechendem Studienmodul erachtet.

\section{Durch offene Lizensierung zur offenen Lernressource (OER)}

Der gesamte Kurs wurde als offene Lernressource (OER) unter CC-BY-Lizenz ${ }^{11}$ erstellt, alle Videos stehen in einer YouTube-Playlist ${ }^{12}$ zur einfachen Weiterverwendung offen bereit und auch die Dokumente im Kurs (Interviews und Foliensätze) können frei heruntergeladen und verwendet werden ${ }^{13}$.

\section{Konsequenzen der Öffnung}

Nachfolgend werden einige Beobachtungen der ersten betreuten Kurserprobung beschrieben, wobei ein Schwerpunkt auf die Aspekte gelegt wird, in denen sich der MOOC durch die Öffnung von klassischen geschlossenen Formaten unterscheidet.

\footnotetext{
Ansprache von potentiellen Teilnehmerinnen und Teilnehmern für die Erprobungsphase Eine für MOOCs nicht ungewöhnliche Herausforderung ist es, die Zielgruppe überhaupt zu erreichen. Anders als im regulären Studium gibt es nicht die bereits eingeschriebenen Studierenden, die diesen Kurs ohnehin belegen müssen. Es gibt schlichtweg keinen vorgezeichneten Weg, auf dem man Berufstätige erreichen kann, die sich

10 Prüfungsstandorte waren die Hochschule Augsburg, die Technische Hochschule Lübeck, die Beuth Hochschule für Technik Berlin, die Frankfurt University of Applied Sciences und die Hochschule Fulda. Darüber hinaus wurden auch die Standorte Ostfalia Wolfenbüttel, Hochschule Emden/Leer, Technische Hochschule Brandenburg und die Allensbach Hochschule Konstanz angeboten, für die es allerdings keine Anmeldungen seitens der MOOC-Teilnehmenden gab.

11 https://creativecommons.org/licenses/by/4.0/deed.de.

12 https://www.youtube.com/watch?v=qkCXdGQsoUE\&index=1\&list=PLQvKY4d5Oi9Nf5IEp_w_0T1gM5ERjW LRV.

13 https://pmooc.oncampus.de/loop/Netzwerksicherheit\#Lernmaterial.
} 
für Fragen der Netzwerksicherheit interessieren könnten, wie z.B. Administratorinnen und Administratoren, Datenschützerinnen und Datenschützer oder Mitarbeiterinnen und Mitarbeiter im Qualitätsmanagement. Zur Gewinnung von Probanden für den ersten betreuten Kursdurchlauf mussten daher andere Wege gesucht werden. Wie im bereits durchgeführten offenen Kurs zum Thema «Projektmanagement» aus dem Projekt pMOOCs wurden in den Social-Media-Kanälen des Instituts für Lerndienstleistung (insb. Facebook, Twitter, YouTube) entsprechende Beiträge und ein Trailer zum Kurs veröffentlicht (siehe hierzu auch ILD 2018).

Als unerwartet erfolgreich erwies sich der Kontakt zum YouTube-Kanal SemperVideo ${ }^{14}$. Der Dozent Andreas Hanemann war auf diesen Kanal aufmerksam geworden und kontaktierte die Betreiberinnen und Betreiber per E-Mail, um sie auf den offenen Kurs aufmerksam zu machen. Am 29.03.2016 stieg die Zahl der Teilnehmenden sprungartig um 1.634 Teilnehmende an (siehe Abbildung 1).

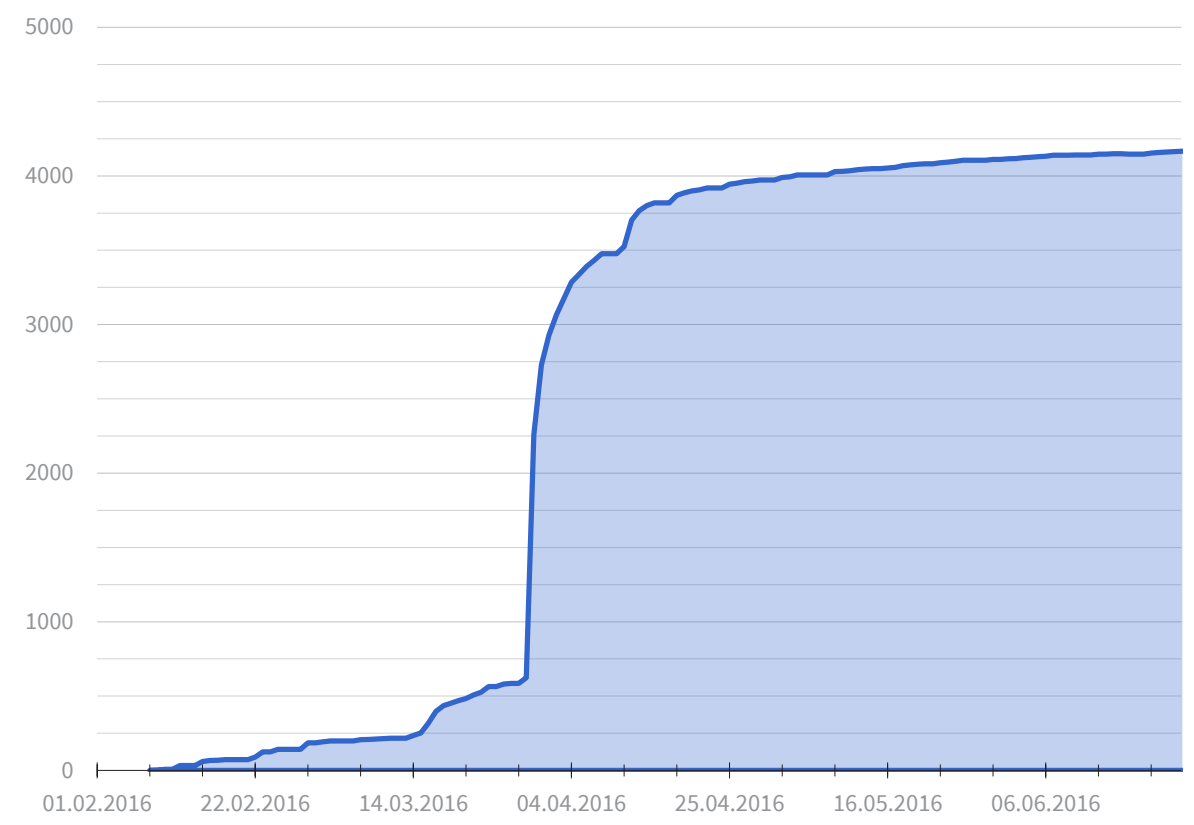

Abb. 1.: Entwicklung der Einschreibungen im nwsMOOC.

Grund hierfür war ein auf SemperVideo veröffentlichtes Video, in dem der Kurs vorgestellt und empfohlen wurde. Der zunächst vermutete ${ }^{15}$ Zusammenhang zwischen dem starken Anstieg und der Ankündigung auf SemperVideo konnte durch die Angaben in der Einstiegsbefragung bestätigt werden (siehe Abbildung 2): von 991 gaben $599(60,44 \%)$ an, über SemperVideo auf den Kurs aufmerksam geworden zu sein.

14 https://www.youtube.com/watch?v=21JRqJreRck.

15 Das Video von SemperVideo in Eigeninitiative erstellt und ohne weitere Absprachen oder Hinweise publiziert. 
Wie sind Sie auf mooin und diesen Kurs aufmweksam geworden?

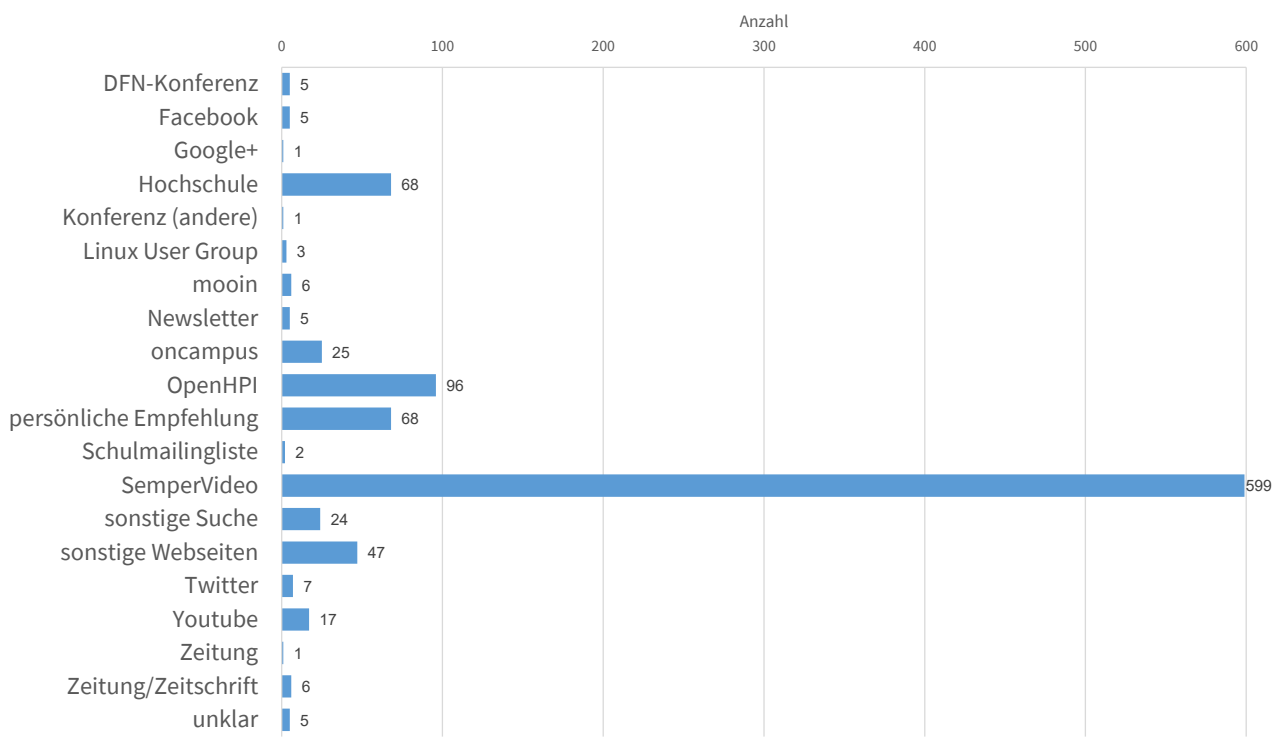

Abb. 2.: Wie sind Sie auf mooin und diesen Kurs aufmerksam geworden? ( $N=991$, da die Antworten im Freitext erfolgten, wurden gleichartige Formulierungen und verschiedene Schreibweisen zusammengefasst).

Eine solche Reichweite in der für den MOOC relevanten Zielgruppe konnte mit dem YouTube-Trailer des Projektes selbst nicht erreicht werden. Die Gegenüberstellung der beiden Videos und YouTube-Kanäle in Tabelle 2 verdeutlicht diese Erkenntnis. Dabei ist anzumerken, dass SemperVideo bereits wenige Tage nach der Veröffentlichung des Videos mehrere tausend Aufrufe vorweisen konnte, während die Klickzahlen für das Video auf oncampusfhl erst über die Zeit zunahmen. Auch wenn in diesem Fall die Betreiberinnen und Betreiber von SemperVideo kein Honorar erhielten, wäre diese Option zur Bekanntmachung offener Angebote offenbar vielversprechend, erfordert aber die Identifikation geeigneter Multiplikatorinnen und Multiplikatoren.

\begin{tabular}{|l|l|l|}
\hline & oncampusthl & SemperVideo \\
\hline Link zur Video-Ankündigung & $\begin{array}{l}\text { https://www.youtube.com/ } \\
\text { watch?v=qkCXdGQsoUE }\end{array}$ & $\begin{array}{l}\text { https://www.youtube.com/ } \\
\text { watch?v=21JRqJreRck }\end{array}$ \\
\hline YouTube-Abonnenten & 6.651 & 286.378 \\
\hline Aufrufe & 12.952 & 28.487 \\
\hline Likes | Dislikes & $34 \mid 3$ & $>1.000 \mid 11$ \\
\hline Kommentare & 28 & 151 \\
\hline
\end{tabular}

Tab. 2.: Vergleich der Ankündigungsvideos (Stand: 03.02.2018). 
Ein Grund hierfür ist eine grundsätzlich andere Nutzung der YouTube-Kanäle: auf dem Kanal oncampusthl werden Videos überwiegend zur Einbettung in Online-Kurse gespeichert, weshalb sie sich in den Themen und Inhalten stark unterscheiden (Erklärvideos aus verschiedenen Bereichen, Trailer für Lernangebote, Vortragsaufzeichnungen, Interviews etc.). Dagegen publiziert SemperVideo regelmässig Videos für eine spezifische Zielgruppe direkt auf YouTube.

\section{Beanspruchung der technischen Infrastruktur}

Auch die technische Infrastruktur wurde durch die Öffnung herausgefordert: der bereitgestellte Testserver kam nach dem Kursstart und insbesondere am Wochenende unter grosse Last (siehe Abbildung 3) und musste um weitere Arbeitsspeichermodule aufgestockt werden.
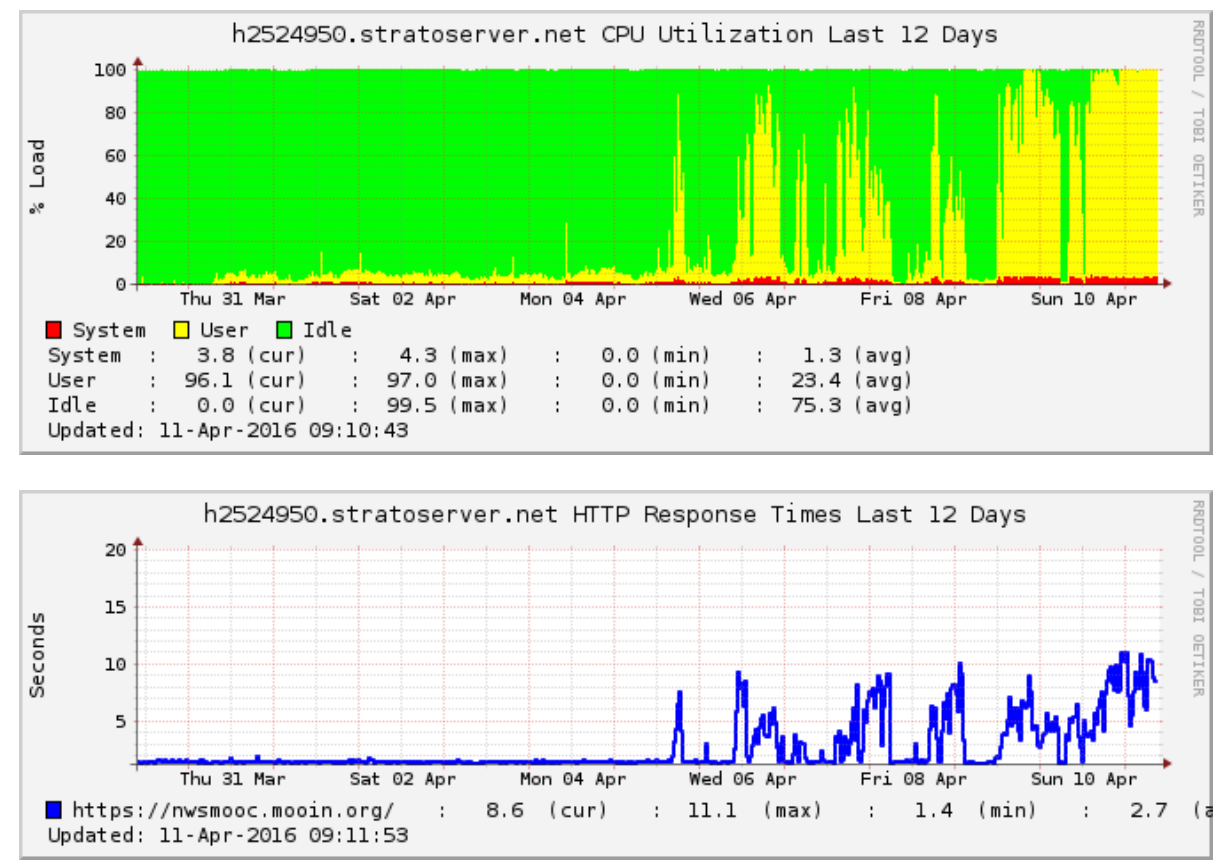

Abb. 3.: Auslastung des bereitgestellten Testservers vom 30.03. bis 11.04.2016.

Einige Testdateien wurden zudem über einen OwnCloud-Server bereitgestellt. Der Service Provider erkannte dabei eine Datei als Virus und forderte dazu auf, diesen zu löschen. Es handelte sich aber um eine bereitgestellte Modifikation der EICARTextdatei, die zum Testen von Antivirusprogrammen heran gezogen werden kann, da sie typische Muster von Schadsoftware enthält ${ }^{16}$. Nachdem dem Service-Provider aber erläutert wurde, dass der Testvirus unschädlich ist und für die Lehre gebraucht wird, durfte er weiterhin über den Server bereitgestellt werden.

16 Siehe auch http://www.eicar.org/86-0-Intended-use.html 


\section{Erkenntnisse aus der Motivation und Dokumentation mittels Digital Badges}

In der Abschlussbefragung gaben 70,8\% ( $\mathrm{N}=113)$ an, dass Sie die Badges als motivierend oder sehr motivierend empfanden. Bei der Frage, was die Teilnehmenden am MOOC begeistert hat, wurden sie in 5 der 93 Freitextantworten positiv genannt, dagegen enthielten sie bei der Frage nach Problemen oder verbesserungswürdigen Aspekten nur einmal eine Nennung («Finde die Badges unnötig», 85 Antworten). Diese Rückmeldungen decken sich auch mit den Evaluationen aus anderen offenen Online-Kursen auf mooin.

Neben der motivierenden Wirkung lassen sich zudem weitere Aspekte an den vergebenen Badges ablesen (siehe Tabelle 3). So wurden bspw. in Kapitel 2 und 3 mehr Aufgaben-Badges für Einsteiger als (die vermeintlich einfacher zu erwerbenden) Video-Badges vergeben. Auch die lobenden Freitextantworten zum Spass an den praktischen Aufgaben sowie die hierdurch entstandene Serverlast lassen zu dem Schluss kommen, dass die kompetenzorientierten Übungsaufgaben den rein wissensüberprüfenden Fragen in den Videos vorgezogen wurden.

\begin{tabular}{|l|l|l|l|l|}
\hline Woche & Video-Badge & $\begin{array}{l}\text { Aufgaben-Badge } \\
\text { Einsteiger }\end{array}$ & $\begin{array}{l}\text { Aufgaben-Badge } \\
\text { Fortgeschrittene }\end{array}$ & $\begin{array}{l}\text { Aufgaben-Badge } \\
\text { Spezialist }\end{array}$ \\
\hline 2 & 355 & 463 & 218 & - \\
& $8,66 \%$ & $11,3 \%$ & $5,32 \%$ & - \\
\hline 3 & 196 & 197 & 99 & - \\
& $4,78 \%$ & $4,87 \%$ & $2,42 \%$ & 48 \\
\hline 4 & 106 & 96 & 66 & $1,17 \%$ \\
\hline 5 & $2,59 \%$ & $2,34 \%$ & $1,61 \%$ & - \\
\hline
\end{tabular}

Tab. 3.: Ausgestellte Badges im nwsMOOC zum Kursende, absolut und prozentual zur Zahl der Teilnehmenden am Kursende ( $n=4099)$.

\section{Möglichkeiten zur Anrechnung erworbener Kompetenzen}

Ein Schwerpunkt lag darauf, Möglichkeiten zur Anrechnung zu schaffen. Von 115 Antworten aus der Abschlussbefragung gab fast ein Drittel $(27,83 \%)$ an, kein Interesse an einer Anrechnung zu haben, $8,7 \%$ war keine Anrechnungsmöglichkeit bekannt und $13,13 \%$ waren noch unsicher. Dagegen wollten $8(6,96 \%)$ an einer Klausur teilnehmen, weitere $14,78 \%$ gaben an, dass sie aufgrund von Termin- oder Ortskonflikten nicht daran teilnehmen könnten. Weitere 11,3\% meinten, zwar keine Prüfungsteilnahme zu planen, aber sich die erworbenen Kompetenzen anderweitig anrechnen zu lassen.

Am 12.07.2016 fand an 5 Standorten der Virtuellen Fachhochschule eine (für die Teilnehmenden optionale) Präsenzklausur statt: an der Hochschule Augsburg, 
der Technischen Hochschule Lübeck, der Beuth Hochschule für Technik Berlin, der Frankfurt University of Applied Siences und der Hochschule Fulda konnten zusätzlich zu den regulären (Online-)Studierenden auch Teilnehmende des MOOCs an der Prüfung teilnehmen. Darüber hinaus standen auch die Standorte Ostfalia Wolfenbüttel, Hochschule Emden/Leer, die Technische Hochschule Brandenburg und die Allensbach Hochschule in Konstanz zur Auswahl, hierfür gab es aber seitens der MOOCTeilnehmenden keinerlei Anmeldung. Der Aufwand zur Organisation dieser ersten offenen Prüfung war relativ hoch, da jeder Standort einzeln angefragt wurde, ob er zusätzlich Plätze für die MOOC-Teilnehmende bereitstellen würde. Bei wiederholter Durchführung einer damit verbundenen Etablierung dieses Vorgehens ist hier aber mit einer deutlichen Aufwandsreduktion zu rechnen.

Die Klausurergebnisse der MOOC-Teilnehmenden unterschieden sich nur um wenige Notenpunkte von denen der regulären Studierenden (siehe Tabelle 4). Wurde die Klausur erfolgreich bestanden, wurde den Teilnehmerinnen und Teilnehmern ein Zertifikat der Technischen Hochschule Lübeck ausgestellt, das eine detaillierte Beschreibung von Workload und Lernergebnissen enthielt. Eine Anrechnung an den Standorten der Virtuellen Fachhochschule für das entsprechende Studienmodul war durch bereits bestehende Anerkennungspraktiken quasi garantiert, über eine Anrechnung anderer Hochschulen entscheidet das jeweilige Prüfungsamt.

\begin{tabular}{|l|l|l|l|l|l|l|l|l|l|l|l|l|l|}
\hline Art & $\boldsymbol{\Sigma}$ & $\mathbf{1 , 0}$ & $\mathbf{1 , 3}$ & $\mathbf{1 , 7}$ & $\mathbf{2 , 0}$ & $\mathbf{2 , 3}$ & $\mathbf{2 , 7}$ & $\mathbf{3 , 0}$ & $\mathbf{3 , 3}$ & $\mathbf{3 , 7}$ & $\mathbf{4 , 0}$ & $\mathbf{5 , 0}$ & $\varnothing$ \\
\hline gesamt & 50 & 6 & 8 & 6 & 4 & 4 & 7 & 3 & 6 & 2 & 2 & 2 & 2,34 \\
\hline MOOC & 20 & 3 & 2 & 2 & 2 & 2 & 1 & 2 & 3 & 1 & 1 & 1 & 2,45 \\
\hline Stud. $^{15}$ & 20 & 3 & 6 & 4 & 2 & 2 & 6 & 1 & 3 & 1 & 1 & 1 & 2,27 \\
\hline
\end{tabular}

Tab. 4.: Vergleich der Leistungen in der Klausur zwischen reinen MOOC-Teilnehmenden und immatrikulierten Online-Studierenden der Virtuellen Fachhochschule.

Um mehr über den Anrechnungserfolg in Erfahrung zu bringen, wurde am 05.12.2016 (also fast 5 Monate nach der Prüfung) erneut ein Fragebogen an die Teilnehmenden der Klausur versendet. Von den 20 Teilnehmenden antworteten 4 Personen, was mit $20 \%$ zwar normalerweise eine gute Rücklaufquote wäre, aufgrund der geringen Anzahl aber wenig generalisierbar ist. Von diesen vier Personen hatte nur eine versucht, die Klausur im Studiengang Wirtschaftsingenieurwesen anzurechnen, allerdings kein entsprechendes Modul identifizieren können. Eine weitere Person gab an, eine Anrechnung noch anzustreben, die anderen beiden dagegen hatten nach eigenen Angaben nie vor, eine Anrechnung zu erwirken.

17 Online-Studierende im Master Medieninformatik (berufsbegleitend). 
Fazit: Quo vadis Hochschullehre?

Mit dem offenen Online-Kurs «Netzwerksicherheit» wurde ein bisher nur den Studierenden vorbehaltenes Hochschulmodul geöffnet und Teilnehmenden unabhängig von Alter, regionaler Verortung oder Hochschulzugangsberechtigung zugängig gemacht. Neben der individuellen Weiterbildung wurde zudem die Anrechnung im Rahmen des Hochschulsystems prinzipiell vorbereitet und eine Möglichkeit zum Übergang in die akademische (Weiter-) Bildung geebnet. Dabei wurden nicht einfach nur Aufzeichnungen der regulären Hochschulformate wie Vorlesungen und Tutorien bereitgestellt, sondern die zu vermittelnden Lerninhalte wurden mit zielgruppengerechten und vor allem kompetenzfördernden digitalen Formaten und Lösungen aufbereitet. Die konsequente Umsetzung als OER ermöglicht zudem eine nachhaltige Weiternutzung des MOOCs. Seit dem Abschluss der betreuten Phase bis Januar 2018 verzeichnete der Kurs 734 neue Teilnehmende ${ }^{18}$. Seitdem wurden weitere offene Kurse im Rahmen des Projekts pMOOCs entwickelt und erprobt ${ }^{19}$.

Letzten Endes werden durch das offene Format aber auch Konzepte infrage gestellt, die in der Präsenzlehre vor allem durch die Infrastruktur und Wirtschaftlichkeit begründet werden. Während die Höhe der (Grund-) Finanzierung u. a. über die Anzahl der immatrikulierten Studierenden einer Hochschule berechnet wird, gibt es aktuell keine Prozesse, die Teilnehmende an offenen Formaten wie MOOCs mit in diese Rechnung einbezieht und honoriert. Derzeit gibt es keinen finanziellen Vorteil (oder wenigstens Ausgleich) für eine Hochschule, Menschen an Lehrveranstaltungen und/oder Prüfungen teilnehmen zu lassen, die nicht oder an anderen Hochschulen immatrikuliert sind.

Auch andere Regelungen werden herausgefordert: So können - anders als bei Klausuren, an der nur immatrikulierte Studierende teilnehmen dürfen - die Anzahl der Versuche bis zum Bestehen nicht valide gezählt werden, was de facto zu einer unendlichen Anzahl an Prüfungsversuchen führt.

Die Öffnung von Hochschulen bringt viele Chancen mit sich, aber sie stellt die Institution Hochschule vor eine Reihe existenzieller Fragen. Allein am Beispiel offener Online-Kurse wird deutlich, dass die Finanzierung von Hochschulen neu gedacht werden muss, wenn deren Angebote auch für Menschen über ihre immatrikulierten Studierenden Hinaus geöffnet werden. Zudem könnte die Bereitstellung des MOOCs Netzwerksicherheit als OER auch dazu führen, dass ihn andere Hochschulen einsetzen und eine Klausur dazu anbieten. Schliesslich stellt dieser Punkt auch das Selbstverständnis von Hochschulen auf die Probe, da sie immer mehr zu Prüfungsorten ¿degradiert) werden könnten, wenn die Lehre selbst online oder an einem anderen Ort ausserhalb der Hochschule stattfindet, und dort zudem noch flexibler, leichter verständlich und unterhaltsamer wahrgenommen wird.

18 Im Monat kamen durchschnittlich 38,6 neue Teilnehmende (zwischen 18 und 68) hinzu.

19 Siehe http://pmooc.oncampus.de/loop/Die_MOOCs_im_Projekt. 
Die bereits im Bologna-Prozess angestrebte Mobilität und Durchlässigkeit des (europäischen) Hochschulsystems hat nun das Potential, mit offenen Angeboten tatsächlich für eine grössere Menschengruppe relevant zu werden. Dabei kommen nun aber auch die oben genannten strukturellen Herausforderungen ans Licht, die bisher in Einzelfallentscheidungen behandelt wurden und kaum institutionelle Beachtung fanden.

\section{Literatur}

BMBF. 2018. «Bund-Länder-Wettbewerb «Aufstieg durch Bildung: offene Hochschulen»». BMBF. 2018. http://www.wettbewerb-offene-hochschulen-bmbf.de/bund-laender-wettbewerbaufstieg-durch-bildung-offene-hochschulen.

Czanderle, Birgit. 2017. «Studierbarkeit. Wesen und Bedeutung für die Akteure im berufsbegleitenden Fernstudium». Zeitschrift Hochschule und Weiterbildung (ZHWB). https://doi. org/10.4119/UNIBI/ZHWB-2017-01-67.

ILD, Hrsg. 2018. «Forschungsfrage 5: Wie lassen sich pMOOCs in unmittelbarer webbasierter Interaktion mit ihren Zielgruppen bedarfsgerecht konzipieren und gestalten?» Institut für Lerndienstleistungen, Technische Hochschule Lübeck. https://pmooc.oncampus.de/mediawiki/images/pmooc.oncampus.de/f/f5/PMOOC_Forschungsfrage_5.pdf.

Jürgens, Alexandra, und Bernd Zinn. 2015. «Nicht-traditionell Studierende in Deutschland Stand der empirischen Forschung und Desiderate». In Beruflich Qualifizierte im Studium: Analysen und Konzepte zum Dritten Bildungsweg, herausgegeben von Uwe Elsholz, 35-56. Bielefeld: W. Bertelsmann Verlag. https://doi.org/10.3278/6004491w.

Lorenz, Anja, Andreas Wittke, Farina Steinert, und Thomas Muschal. 2015. «Massive Open Online Courses als Teil der Hochschulstrategie». In Digitale Medien und Interdisziplinarität: Herausforderungen, Erfahrungen, Perspektiven, herausgegeben von Nicolae Nistor und Sabine Schirlitz, 68:102-112. Medien in der Wissenschaft. München: Waxmann. http://2015. gmw-online.de/102/.

Wannemacher, Klaus. 2014. «Digitale Weiterbildungsangebote an deutschsprachigen Hochschulen». In Postgraduale Bildung mit digitalen Medien: Fallbeispiele aus den sächsischen Hochschulen, herausgegeben von Helge Fischer und Thomas Köhler, 65:13-25. Medien in der Wissenschaft. Münster, New York: Waxmann. 